\title{
ESTUDO CLIINICO DO QUADRIL NÃO TRATADO NA TETRAPARESIA ESPÁSTICA
}

\author{
CLINICAL STUDY OF UNTREATED HIP IN SPASTIC TETRAPARESIS
}

Fernanda Catena', Eduardo Ramalho de Moraes ${ }^{1}$, Andre Vitor Kerber Cavalcanti Lemos', Patrícia Corey Yamane², Francesco Camara Blumetti ${ }^{2}$, Eiffel Tsuyoshi Dobashi ${ }^{3}$, José Antonio Pinto ${ }^{4}$

\section{RESUMO}

Objetivo: Avaliar quadris de pacientes com tetraparesia espástica considerando a gravidade das deformidades articulares, a idade e a escoliose. Métodos: Realizamos um estudo descritivo transversal de 40 pacientes (um a 17 anos); 21 (52,5\%) do sexo feminino e $19(47,5 \%)$ do masculino em pacientes da Associação Cruz Verde; $19(47,5 \%)$ apresentaram prematuridade, $26(65,0 \%)$ anóxia, quatro (10,0\%) meningite, $10(25,0 \%)$ hidrocefalia e $15(37,5 \%)$ microcefalia; $38(95,0 \%)$ apresentavam espasticidade, um (2,5\%) atetose e um (2,5\%) o padrão misto; 28 (70,0\%) apresentavam tetraparesia e $12(30,0 \%)$ dupla hemiparesia. Nenhum paciente deambulava, 38 (95,0\%) foram classificados como GFMCS V e dois $(5,0 \%)$ como IV. Consideramos dois grupos, conforme a necessidade ou não do tratamento cirúrgico dos quadris pelo risco de luxação e dor. Utilizamos os testes de Thomas, abdução brusca e Nelaton-Galeazzi. A escoliose foi avaliada pela simetria do tronco e gibosidade. Resultados: Observamos correlação entre a escoliose e a positividade do teste da abdução brusca. Não houve correlação entre a idade e a contratura dos quadris. Não houve diferença entre os tipos topográficos e a contratura do quadril. Conclusões: Não encontramos correlação entre a idade e o grau de contratura articular; pacientes com escoliose apresentaram maior comprometimento da abdução do quadril.

Descritores - Quadril; Paralisia Cerebral; Contratura; Escoliose

\section{ABSTRACT}

Objective: To evaluate CP patients considering the hip joint and analyzing the relation between severity, age and scoliosis. Methods: A transversal descriptive study was performed in 40 patients (1 to 17 y.o.), 21 (52,5\%) female and 19 (47,5\%) male from Cruz Verde Association; 19 (47,5\%) had history of prematurity; 26 (65,0\%) with anoxia; meningitis in 4 (10\%), hydrocephalus in 10(25,0\%); microcephalus in 15 (37,5\%). From the total 38 (95,0\%) presented spasticity, 1 (2,5\%) athetoid and 1 (2,5\%) mixed. According to topographic classification 28 (70,0\%) presented tetraparesis and 12 (30,0\%) double hemiparesis. All of them are non-ambulators and 38 (95,0\%) were classified as GFMCS V and 2 (5,0\%) were level IV. The patients were divided in two groups (with or without indications for hip surgical treatment). The hips were analysed by Thomas, abduction, and Nelaton-Galeazzi tests. The scoliosis was clinically analysed considering trunk symmetry and dorsal hump. Results: We observed positive correlation between the patients' scoliosis and hip flexion contracture. There was no difference between the patients' age and and hip flexion contracture. There was no difference between topographic types and hip contractures. Conclusions: We did not find positive correlation between patients' age and hip contractures; patients with scoliosis present worse hip abduction compromise.

Keywords - Hip; Cerebral Palsy; Contracture; Scoliosis

\section{INTRODUÇÃO}

A paralisia cerebral (PC) é definida como um transtorno permanente da evolução do movimento e da postura, causando limitação da atividade atribuída a um distúrbio não progressivo afetando o sistema nervoso central imaturo $^{(1)}$.

É importante salientar que, apesar da lesão cerebral permanecer estática em seu tamanho, as alterações

1 - Médico Residente do Terceiro Ano do Curso de Ortopedia e Traumatologia da Universidade Federal de São Paulo - Unifesp/EPM - São Paulo, SP, Brasil.

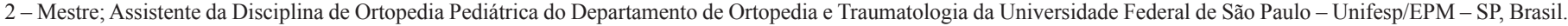

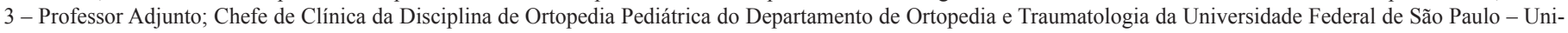
fesp/EPM - SP, Brasil.

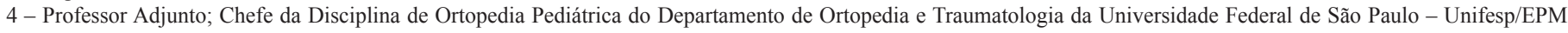
SP, Brasil..

Trabalho realizado na Associação Cruz Verde.

Correspondência: Rua Borges Lagoa, 783, $5^{\circ}$ andar, Vila Clementino - 04038-032 - São Paulo, SP. E-mail: fecatena@hotmail.com

Trabalho recebido para publicação: 24/08/2011, aceito para publicação: 31/08/2011

Os autores declaram inexistência de conflito de interesses na realização deste trabalho / The authors declare that there was no conflict of interest in conducting this work 
musculoesqueléticas resultantes irão invariavelmente progredir em decorrência da espasticidade, da fraqueza muscular e da deficiência do crescimento longitudinal do esqueleto.

As articulações apresentam diminuição do arco de movimento, pois o músculo hipertônico, em razão do reflexo de estiramento, determina uma resistência opositora que progressivamente vai aumentando ${ }^{(2)}$. Logo as alterações anatômicas decorrentes da PC dependem diretamente do grau de comprometimento neurológico, do desenvolvimento motor e da idade do paciente ${ }^{(1,3-5)}$.

Particularmente, a deformidade do quadril é bastante frequente, sendo reconhecida em $25 \%$ a $75 \%$ dos pacientes, tem etiologia multifatorial e está diretamente relacionada com a gravidade e a complexidade do comprometimento neurológico. Portanto, esta é mais prevalente nos pacientes tetraparéticos e nos não deambuladores ${ }^{(6-12)}$.

O comprometimento deste segmento é inicialmente postural, mas pode evoluir até que as deformidades se tornem fixas (subluxação e luxação, persistência da anteversão femoral, rotação medial das coxas e quadris em ventania $)^{(13)}$.

Estas deformidades determinam uma diminuição da mobilidade articular, dificuldades de posicionamento, a diminuição e até a perda da capacidade de sentar-se, problemas na higiene perineal e o aparecimento de úlceras de pressão( ${ }^{(8,10,12,14-16)}$. Além disso, a posição em adução do quadril contribui para o desenvolvimento de obliquidade pélvica e subsequente escoliose ${ }^{(10-12)}$.

Como o impacto socioeconômico do tratamento desta população é bastante elevado, um dos paradigmas a ser considerado para esta afecção deve focar a prevenção. Estas medidas visam impedir a instalação destas deformidades determinando uma melhor condição de vida.

Este estudo foi elaborado com o objetivo de avaliar pacientes portadores de PC grave, considerando especialmente as alterações da articulação do quadril e analisando se há relação entre a gravidade das deformidades com a idade dos pacientes e a associação com escoliose. Os resultados obtidos poderão determinar melhor compreensão sobre as alterações desta articulação em que serão discutidos os benefícios das terapias empregadas para prevenção e tratamento das deformidades.

\section{CASUÍSTICA E MÉTODOS}

Este estudo foi avaliado e aprovado pelo Comitê de Ética e Pesquisa da Unifesp/EPM e está registrado com o número CEP 0285/11.

Foi realizado um estudo descritivo transversal em pacientes com PC grave institucionalizados na Associação Cruz Verde, especializada em cuidar de pessoas portadoras desta doença.

Todos os pacientes internados nesta instituição foram incluídos para a execução deste estudo a partir da análise dos seus prontuários. Como critérios de inclusão consideramos os pacientes portadores da encefalopatia crônica, portadores da tetraparesia e dupla hemiparesia, não deambuladores, sem tratamento cirúrgico prévio. Foram excluídos os que apresentavam outro tipo de comprometimento e/ou deambuladores.

Analisamos clinicamente 40 pacientes de um a 17 anos, sendo $21(52,5 \%)$ do sexo feminino e $19(47,5 \%)$ do masculino. Com relação à idade, quatro $(10,0 \%)$ pacientes tinham de um a quatro anos, cinco $(12,5 \%)$ de cinco a oito anos, $12(30,0 \%)$ de nove a 12 anos e 19 $(47,5 \%)$ de 13 a 17 anos. Quanto ao grupo étnico, 22 $(55,0 \%)$ eram brancos, 14 (35,0\%) não brancos e quatro $(10,0 \%)$, negros.

Observamos pela análise dos prontuários que 19 (47,5\%) pacientes tinham história de prematuridade. Anóxia cerebral foi verificada em 26 (65,0\%), meningite em quatro (10\%), hidrocefalia em $10(25 \%)$ e microcefalia em 15 (37,5\%) dos casos estudados.

Os pacientes foram classificados de acordo com o tipo de manifestação neurológica em cinco tipos ${ }^{(17)}$.

1. Espástico: quando há aumento do reflexo de estiramento. 2. Discinético: ocorrem movimentos anormais que são percebidos quando o paciente inicia a movimentação. Quando o mesmo é examinado na posição supina e completamente relaxado, geralmente percebemos que o arco de movimento passivo é completo e o tônus encontra-se muscular diminuído. Neste caso, dois grupos podem ser encontrados:

a) Coreoatetoico: ocorre uma movimentação maciça involuntária e sem propósito do membro avaliado, que, na fase motora inicial, uma extremidade leva ao movimento de outros grupos musculares não correlatos em outras regiões.

b) Distônico: ocorre um desvio do tônus basal, induzido pelo movimento. Tipicamente, as crianças com este tipo de comprometimento assumem e mantêm posturas distorcidas e padrões estereotipados. Estes podem ser confundidos com os tipos espásticos, principalmente quando hipertônicos.

3. Atáxico: há alteração na coordenação voluntária dos movimentos devido à dissinergia muscular. É causada por lesão cerebelar, o que se verifica pela frequente as- 
sociação com marcha de base alargada e tremor intencional leve (dismetria).

4. Mista: há envolvimento tanto do sistema piramidal como do extrapiramidal. Geralmente, este tipo ocorre nas lesões cerebrais mais graves, estando mais frequentemente associado à quadriplegia.

5. Hipotônico: caracteriza-se pela fraqueza e hipotonia muscular com reflexos tendinosos presentes. Esta forma cursa com alterações graves prejudicando o equilíbrio do tronco para sentar, posicionar o segmento cefálico e dificuldade para a comunicação.

A grande maioria dos pacientes avaliados (38 pacientes, 95,0\%) apresentava espasticidade, um (2,5\%) foi classificado como atetoide e outro $(2,5 \%)$ como misto.

Segundo a classificação topográfica de $\operatorname{Minear}^{(18)}, 28$ (70,0\%) pacientes foram classificados como tetraparéticos e $12(30,0 \%)$, com dupla hemiparesia.

A classificação funcional pelo Sistema de Classificação da Função Motora Grosseira (GMFCS), que se baseia na capacidade motora voluntária enfatizando particularmente o sentar (controle do tronco) e o andar, também foi aplicada em nossa casuística. Como um dos critérios era não ser deambulador, 38 (95,0\%) foram classificados como nível $\mathrm{V}$ e dois $(5,0 \%)$, como nível IV.

Os pacientes foram divididos em dois grupos de acordo com a necessidade do tratamento cirúrgico para os quadris (dor e/ou risco de luxação) e dos que não receberam nenhuma indicação de tratamento cirúrgico.

Os quadris de todos os pacientes foram avaliados utilizando-se o teste de Thomas para verificar a presença ou não da limitação da extensão do quadril. Doenças intra-articulares, frequentemente, desencadeiam resposta reflexa antálgica com flexão desta articulação. Eventualmente, esta limitação não é percebida porque é compensada pela inclinação da pelve. A manobra de Thomas elimina a atitude compensatória da pelve e evidência a posição real do quadril no decúbito dorsal. $\mathrm{O}$ ângulo formado pela superfície plana da maca e a coxa do paciente foi medido em todos os pacientes com auxílio de goniômetro.

Os pacientes também foram avaliados pelo teste de abdução brusca do quadril, pelo qual avaliamos a presença da contratura em adução do quadril pela espasticidade. Com o paciente em decúbito supino é realizada uma rápida abdução do quadril partindo da posição neutra e o ângulo de abdução é medido.

O teste de Nelaton-Galeazzi foi aplicado para verificar a diferença de comprimento nos membros inferiores ${ }^{(19)}$.
Avaliamos a escoliose na população estudada pela presença ou não dos seguintes parâmetros clínicos: assimetria do tronco e gibosidade. Os indivíduos com equilíbrio do tronco foram examinados em posição ortostática pela vista posterior com a ajuda de um ou dois auxiliares. Os indivíduos sem capacidade para assumir o ortostatismo foram avaliados sentados com os membros pendentes e mantidos nesta posição com a ajuda de um ou dois auxiliares.

Após a coleta dos dados foi feita uma análise estatística descritiva e não paramétrica entre os dois grupos de pacientes. Foram usadas as correlações de Spearman para avaliar a relação entre idade e as variáveis obtidas no exame do quadril. O teste de Mann-Whitney foi utilizado para avaliar a relação entre os testes clínicos do quadril, a ocorrência de escoliose, a classificação topográfica dos pacientes e a gravidade do comprometimento coxofemoral.

\section{RESULTADOS}

No grupo de pacientes que receberam alguma indicação de tratamento cirúrgico, o ângulo mensurado pela execução do teste de Thomas do quadril esquerdo variou de $0^{\circ}$ a $80^{\circ}$ com média de $14,42^{\circ}$. Para o quadril direito, o valor mínimo foi de $0^{\circ}$ e o valor máximo foi de $80^{\circ}$, com média de $32,5^{\circ}$. O valor mínimo do ângulo de abdução brusca foi de $0^{\circ}$ e o máximo foi de $60^{\circ}$, com média de $17,14^{\circ}$.

No grupo de pacientes em que não havia necessidade iminente de tratamento cirúrgico, o valor mínimo do teste de Thomas do quadril esquerdo foi de $0^{\circ}$ e o valor máximo foi de $90^{\circ}$, com média de $17,31^{\circ}$. Para o quadril direito, o valor mínimo foi de $0^{\circ}$ e o valor máximo foi de $65^{\circ}$, com média de 26,07 . O valor mínimo do ângulo de abdução brusca foi de $0^{\circ}$ e o máximo foi de $50^{\circ}$, com média de $24,62^{\circ}$.

A Tabela 1 demonstra os resultados do coeficiente de correlação de Spearman entre as medidas do teste de Thomas, do ângulo de abdução brusca e a idade dos pacientes estudados.

Elaboramos a Tabela 2 para demonstrar o resultado do teste de Mann-Whitney para a correlação entre as medidas do teste de Thomas e do ângulo de abdução brusca entre os grupos.

A Tabela 3 apresenta o resultado do teste de MannWhitney para a correlação entre as medidas do teste de Thomas, o ângulo de abdução brusca e a presença de escoliose. 
Tabela 1 - Resultado das correlações de Spearman entre idade e medidas do quadril.

\begin{tabular}{c|c|c|c|c}
\hline $\begin{array}{c}\text { Indicação de } \\
\text { tratamento } \\
\text { cirúrgico }\end{array}$ & Variável & Correlação & $\mathbf{N}$ & $\mathbf{p}$ \\
\hline \multirow{5}{*}{ Não } & $\begin{array}{c}\text { Quadril E Thomas } \\
\text { (graus) }\end{array}$ & 0,163 & 26 & 0,427 \\
\cline { 2 - 5 } & $\begin{array}{c}\text { Quadril D Thomas } \\
\text { (graus) }\end{array}$ & $-0,102$ & 26 & 0,619 \\
\cline { 2 - 5 } & $\begin{array}{c}\text { Abdução brusca } \\
\text { (graus) }\end{array}$ & $-0,192$ & 26 & 0,348 \\
\hline \multirow{5}{*}{ Sim } & $\begin{array}{c}\text { Quadril E Thomas } \\
\text { (graus) }\end{array}$ & 0,698 & 14 & 0,005 \\
\cline { 2 - 5 } & $\begin{array}{c}\text { Quadril D Thomas } \\
\text { (graus) }\end{array}$ & 0,472 & 14 & 0,088 \\
\cline { 2 - 5 } & $\begin{array}{c}\text { Abdução brusca } \\
\text { (graus) }\end{array}$ & $-0,219$ & 14 & 0,451 \\
\hline \multirow{5}{*}{ Total } & $\begin{array}{c}\text { Quadril E Thomas } \\
\text { (graus) }\end{array}$ & 0,326 & 40 & 0,040 \\
\cline { 2 - 5 } & $\begin{array}{c}\text { Quadril D Thomas } \\
\text { (graus) }\end{array}$ & 0,1 & 40 & 0,540 \\
\cline { 2 - 5 } & $\begin{array}{c}\text { Abdução brusca } \\
\text { (graus) }\end{array}$ & $-0,173$ & 40 & 0,287 \\
\hline
\end{tabular}

Tabela 3 - Resultado dos testes de Mann-Whitney.

\begin{tabular}{|c|c|c|c|c|c|}
\hline Casos & I vasc. & I. nervosa & $\begin{array}{c}\text { Sínd. } \\
\text { compart. }\end{array}$ & $\begin{array}{l}\text { resultado } \\
\text { funcional }\end{array}$ & $\begin{array}{l}\text { Deformidade } \\
\text { angular } \\
\text { residual }\end{array}$ \\
\hline 1 & $\mathrm{~N}$ & $\begin{array}{c}\text { Interósseo } \\
\text { post }\end{array}$ & $\mathrm{N}$ & Normal & Ausente \\
\hline 2 & $\mathrm{~N}$ & $\mathrm{~N}$ & $\mathrm{~N}$ & Normal & Ausente \\
\hline 3 & $\mathrm{~N}$ & $\mathrm{~N}$ & $\mathrm{~N}$ & Normal & Ausente \\
\hline 4 & $\mathrm{~N}$ & $\mathrm{~N}$ & $\mathrm{~N}$ & Normal & Ausente \\
\hline 5 & $\mathrm{~N}$ & $\mathrm{~N}$ & $\mathrm{~N}$ & Normal & Ausente \\
\hline 6 & $\mathrm{~N}$ & $\mathrm{~N}$ & $\mathrm{~N}$ & Normal & Ausente \\
\hline 7 & $\mathrm{~N}$ & $\mathrm{~N}$ & $\mathrm{~N}$ & Normal & Ausente \\
\hline 8 & $\mathrm{~N}$ & $\mathrm{~N}$ & $\mathrm{~N}$ & Normal & Ausente \\
\hline 9 & $\mathrm{~N}$ & $\mathrm{~N}$ & $\mathrm{~N}$ & Normal & Ausente \\
\hline 10 & $\mathrm{~N}$ & Mediano & $\mathrm{N}$ & Normal & Ausente \\
\hline 11 & $\mathrm{~N}$ & $\mathrm{~N}$ & $\mathrm{~N}$ & Normal & Ausente \\
\hline 12 & $\mathrm{~N}$ & $\mathrm{~N}$ & $S$ & Normal & Ausente \\
\hline 13 & $\mathrm{~N}$ & $\mathrm{~N}$ & $\mathrm{~N}$ & Normal & Varo 5 graus \\
\hline 14 & $\mathrm{~N}$ & $\mathrm{~N}$ & $\mathrm{~N}$ & Normal & Ausente \\
\hline 15 & $\mathrm{~N}$ & $\mathrm{~N}$ & $\mathrm{~N}$ & Normal & Ausente \\
\hline
\end{tabular}

Tabela 2 - Resultado dos testes de Mann-Whitney.

\begin{tabular}{|c|c|c|c|c|c|c|c|c|}
\hline Variável & $\begin{array}{l}\text { Indicação de } \\
\text { tratamento } \\
\text { cirúrgico }\end{array}$ & Média & DP & Mediana & Mínimo & Máximo & $\mathrm{N}$ & $p$ \\
\hline \multirow{2}{*}{$\begin{array}{c}\text { Quadril E Thomas } \\
\text { (graus) }\end{array}$} & Não & 17,31 & 26,77 & 0 & 0 & 90 & 26 & \multirow{2}{*}{0,292} \\
\hline & Sim & 26,07 & 28,90 & 15 & 0 & 80 & 14 & \\
\hline \multirow{2}{*}{$\begin{array}{c}\text { Quadril D Thomas } \\
\text { (graus) }\end{array}$} & Não & 14,42 & 19,71 & 0 & 0 & 65 & 26 & \multirow{2}{*}{0,036} \\
\hline & Sim & 32,50 & 28,94 & 35 & 0 & 80 & 14 & \\
\hline \multirow{2}{*}{$\begin{array}{c}\text { Abdução brusca } \\
\text { (graus) }\end{array}$} & Não & 24,62 & 19,13 & 20 & 0 & 50 & 26 & \multirow{2}{*}{0,266} \\
\hline & Sim & 17,14 & 18,58 & 12,5 & 0 & 60 & 14 & \\
\hline
\end{tabular}

A Tabela 4 mostra os resultados do teste de MannWhitney para a correlação entre as medidas do teste de Thomas, do ângulo de abdução brusca e a classificação topográfica.

\section{DISCUSSÃO}

De acordo com a literatura, a deformidade no quadril que mais cedo aparece nos pacientes portadores de PC é a flexão associada à adução. Seu tratamento deve ser iniciado o mais precocemente possível por meio de fisioterapia específica pela aplicação de manobras para alongamento dos músculos adutores e flexores do quadril. Devem ser também adotadas medidas cautelares para a melhora da postura desses pacientes, procurando manter sempre os membros inferiores em abdução.

Quando o desequilíbrio muscular se mantém, a despeito do tratamento fisioterapêutico adequado, na maioria dos casos é necessária uma abordagem cirúrgica mediante a utilização de tenotomias dos músculos adutores e do iliopsoas, com o objetivo de reequilibrar a musculatura atuante no quadril ${ }^{(20)}$.

Diversos autores mostram que mesmo os pacientes adequadamente tratados e que foram submetidos a uma apropriada liberação das partes moles apresentam progressão da deformidade do quadril ${ }^{(21-24)}$. Em contrapartida, existem os que advogam que a tenotomia dos adutores representa um procedimento altamente eficaz na mudança da trajetória da displasia coxofemoral espástica $^{(3)}$ salientando as vantagens dessa técnica cirúrgica no controle do deslocamento do quadril ${ }^{(25-29)}$.

Entretanto, apontamos que este procedimento efetuado isoladamente não apresenta a eficácia profilática quando consideramos a população de indivíduos não deambuladores, como observamos no trabalho de Kalen e Bleck, que obtiveram $64 \%$ de maus resultados ${ }^{(5)}$. 
Tabela 4 - Resultado dos testes de Mann-Whitney.

\begin{tabular}{|c|c|c|c|c|c|c|c|c|c|}
\hline $\begin{array}{l}\text { Indicação de } \\
\text { tratamento } \\
\text { cirúrgico }\end{array}$ & Variável & Classifição topográfica & Média & DP & Mediana & Mínimo & Máximo & $\mathbf{N}$ & $\mathbf{p}$ \\
\hline \multirow{6}{*}{ Não } & \multirow{2}{*}{$\begin{array}{c}\text { Quadril E Thomas } \\
\text { (graus) }\end{array}$} & Dupla hemiparesia & 12,14 & 16,55 & 5 & 0 & 45 & 7 & \multirow{2}{*}{0,955} \\
\hline & & Tetraparesia & 19,21 & 29,83 & 0 & 0 & 90 & 19 & \\
\hline & \multirow{2}{*}{$\begin{array}{c}\text { Quadril D Thomas } \\
\text { (graus) }\end{array}$} & Dupla hemiparesia & 12,86 & 23,78 & 0 & 0 & 65 & 7 & \multirow{2}{*}{0,692} \\
\hline & & Tetraparesia & 15,00 & 18,71 & 0 & 0 & 55 & 19 & \\
\hline & \multirow{2}{*}{$\begin{array}{c}\text { Abdução brusca } \\
\text { (graus) }\end{array}$} & Dupla hemiparesia & 22,86 & 19,97 & 20 & 0 & 50 & 7 & \multirow{2}{*}{0,866} \\
\hline & & Tetraparesia & 25,26 & 19,33 & 20 & 0 & 50 & 19 & \\
\hline \multirow{6}{*}{ Sim } & \multirow{2}{*}{$\begin{array}{c}\text { Quadril E Thomas } \\
\text { (graus) }\end{array}$} & Dupla hemiparesia & 22,00 & 33,47 & 10 & 0 & 80 & 5 & \multirow{2}{*}{0,797} \\
\hline & & Tetraparesia & 28,33 & 27,95 & 30 & 0 & 70 & 9 & \\
\hline & \multirow{2}{*}{$\begin{array}{c}\text { Quadril D Thomas } \\
\text { (graus) }\end{array}$} & Dupla hemiparesia & 23,00 & 25,88 & 10 & 0 & 60 & 5 & \multirow{2}{*}{0,518} \\
\hline & & Tetraparesia & 37,78 & 30,63 & 45 & 0 & 80 & 9 & \\
\hline & \multirow{2}{*}{$\begin{array}{c}\text { Abdução brusca } \\
\text { (graus) }\end{array}$} & Dupla hemiparesia & 20,00 & 18,37 & 20 & 5 & 50 & 5 & \multirow{2}{*}{0,518} \\
\hline & & Tetraparesia & 15,56 & 19,60 & 10 & 0 & 60 & 9 & \\
\hline \multirow{6}{*}{ Total } & \multirow{2}{*}{$\begin{array}{c}\text { Quadril E Thomas } \\
\text { (graus) }\end{array}$} & Dupla hemiparesia & 16,25 & 24,13 & 7,5 & 0 & 80 & 12 & \multirow{2}{*}{0,864} \\
\hline & & Tetraparesia & 22,14 & 29,04 & 2,5 & 0 & 90 & 28 & \\
\hline & \multirow{2}{*}{$\begin{array}{c}\text { Quadril D Thomas } \\
\text { (graus) }\end{array}$} & Dupla hemiparesia & 17,08 & 24,07 & 7,5 & 0 & 65 & 12 & \multirow{2}{*}{0,656} \\
\hline & & Tetraparesia & 22,32 & 25,07 & 17,5 & 0 & 80 & 28 & \\
\hline & \multirow{2}{*}{$\begin{array}{c}\text { Abdução brusca } \\
\text { (graus) }\end{array}$} & Dupla hemiparesia & 21,67 & 18,51 & 20 & 0 & 50 & 12 & \multirow{2}{*}{0,893} \\
\hline & & Tetraparesia & 22,14 & 19,60 & 17,5 & 0 & 60 & 28 & \\
\hline
\end{tabular}

Reforçamos a ideia de que o atraso na indicação cirúrgica deve contribuir substancialmente para os altos índices de insucesso, e assim o caráter preventivo desta operação perderia seu fundamento.

Agregada ao desequilíbrio muscular e do deficiente equilíbrio do tronco, a ausência da capacidade de deambular atua como mais um fator agravante, pois o colo femoral mantém sua configuração anatômica em valgo e em anteversão e o teto acetabular vai inclinando-se progressivamente, tornando-se displásico ${ }^{(20)}$.

A análise estatística dos resultados obtidos na nossa amostra evidenciou a correlação estatisticamente significativa nas seguintes condições. Inicialmente, observamos uma correlação positiva entre a idade do paciente com indicação de tratamento cirúrgico e um maior grau de contratura em flexão do quadril esquerdo evidenciado pelo teste de Thomas $(\mathrm{p}=0,040)$.

Um dos escopos de nosso estudo sugere haver relação entre a presença de escoliose e um pior grau de contratura em adução dos quadris $(\mathrm{p}=0,018)$. Observamos que os pacientes com escoliose apresentaram um menor ângulo de abdução brusca do quadril. A associação de um menor ângulo de abdução brusca do quadril nos pacientes com escoliose é um dado novo e necessita de uma análise mais detalhada desse fato. A relação entre a obliquidade pélvica e a presença de escoliose foi inicialmente descrita por Lonstein e Beck ${ }^{(1)}$. Entretanto, essa contratura não poderia influenciar diretamente a obliquidade da cintura pélvica, apontada na literatura como possível causa de deformidades da coluna nestes pacientes. Além disso, o fato de os indivíduos estudados não apresentarem controle do tronco e permanecerem deitados a maior parte do tempo coloca em dúvida a veracidade desta relação, como o observado na maioria dos casos de nossa amostra. O maior grau de espasticidade estaria diretamente relacionado com um maior comprometimento corporal destes pacientes.

De acordo com Reimers ${ }^{(30)}$, as contraturas nos pacientes com PC espástica se desenvolvem em duas etapas. No primeiro estágio, observamos uma contratura funcional, assim chamada por evidenciarmos as alterações motoras somente quando o paciente executa movimento. No segundo estágio, as contraturas articulares tornam-se estruturadas pela presença de alterações morfológicas articulares e das estruturas ligamentares ${ }^{(13)}$.

É consenso na literatura que o tratamento ideal dos pacientes no primeiro estágio seja preventivo, caso estes se apresentem clinicamente com limitação da mobilidade em abdução e flexão, também conhecidos como "quadris em risco". Este tratamento baseia-se em: fisioterapia; uso de órteses de posicionamento; drogas para diminuir o tônus, como Diazepam, Baclofeno e Dantrolene; alcoolização, fenolização ou, mais recentemente, a injeção de toxina botulínica ${ }^{(18)}$ nos grupos musculares 
espásticos. Entre as opções cirúrgicas, podem ser realizados bloqueios de raízes nervosas lombares (rizotomia) ${ }^{(31)}$ ou tenotomias mediante a liberação da musculatura espástica causadora do desequilíbrio muscular. Concordamos com esta assertiva; contudo, nem todos os recursos utilizáveis estão disponíveis em nosso meio.

A falha terapêutica determina, em um momento ulterior, a necessidade de utilizarmos tratamentos cirúrgicos complexos que visam o restabelecimento anatômico e funcional dos segmentos comprometidos, associando a liberação de partes moles às osteotomias pélvicas e femorais $^{(6,7,10-12,32,33)}$.

O ônus pela falta de acesso ao tratamento adequado incrementa os riscos clínicos, como o de escaras, cardíacos, respiratórios, intestinais, infecções etc. Os transtornos do aparelho locomotor interferem na postura adequada e na higiene. Além do mais ressaltamos que os quadris de pacientes portadores de PC podem cursar com dor, o que prejudica ainda mais os cuidados destes. Acreditamos que ainda são necessários mais estudos sobre esta complexa entidade.

\section{CONCLUSÕES}

Não encontramos correlação estatística significante entre a idade dos pacientes estudados e o grau de contratura do quadril. Os pacientes com escoliose apresentaram maior comprometimento da abdução do quadril pela positividade do teste de abdução brusca $(p=0,018)$.

\section{REFERÊNCIAS}

1. Lonstein JE, Beck K. Hip dislocation and subluxation in cerebral palsy. J Pediatr Orthop. 1986;6(5):521-6.

2. De Paula A. Deformidades estáticas, dinâmicas e mistas na paralisia cerebral: reflexões sobre a semiologia e nomenclatura. Acta Ortop Bras. 1996;4(1):42,

3. Pinto J A, Masiero D, Milani C, Ishida A, Dobashi E, Saad E A. Avaliação tomográfica em quadris de pacientes diplégicos espásticos. Rev Bras Ortop. 1996;31(12):1013-8.

4. Griffiths GJ, Evans KT, Roberts GM, Lloyd KN. The radiology of the hip joints and pelvis in cerebral palsy. Clin Radiol. 1977;28(2):187-91.

5. Gamble JG, Rinsky LA, Bleck EE. Established hip dislocations in children with cerebral palsy. Clin Orthop Relat Res. 1990;(253):90-9.

6. Lyne ED, Katcherian DA. Slotted acetabular augmentation in patients with neuromuscular disorders. J Pediatr Orthop. 1988;8(3):278-84.

7. Herndon WA, Bolano L, Sullivan JA. Hip stabilization in severely involved cerebral palsy patients. J Pediatr Orthop. 1992;12(1):68-73.

8. Bagg MR, Farber J, Miller F. Long-term follow-up of hip subluxation in cerebral palsy patients. J Pediatr Orthop. 1993;13(1):32-6.

9. Miller F, Girardi H, Lipton G, Ponzio R, Klaumann M, Dabney KW. Reconstruction of the dysplastic spastic hip with peri-ilial pelvic and femoral osteotomy followed by immediate mobilization. J Pediatr Orthop. 1997;17(5):592-602.

10. Fujiwara M, Basmajian JV, Iwamoto M. Hip abnormalities in cerebral palsy: radiological study. Arch Phys Med Rehabil. 1976;57(6):278-81.

11. Herring AJ. Disorders of the brain. In: Tadjian's Pediatric Orthopaedics. 3th ed. Philadelphia: Saunders; 2002. p.1121-248.

12. Miller F. Cerebral palsy. New York: Springer Science-Business Media: 2005. p.523-666

13. Sathler M, Miotomia seletiva de flexores do quadril em pacientes com paralisia cerebral Análise retrospectiva a longo prazo. Rev Bras Ortop. 1994; 29(5):345-50,

14. Houkom JA, Roach JW, Wenger DR, Speck G, Herring JA, Norris EN. Treatment of acquired hip subluxation in cerebral palsy. J Pediatr Orthop. 1986;6(3):285-90.

15. Kalen V, Bleck EE. Prevention of spastic paralytic dislocation of the hip. Dev Med Child Neurol. 1985;27(1):17-24.

16. Peterson HA, Klassen RA, McLeod RA, Hoffman AD. The use of computerised tomography in dislocation of the hip and femoral neck anteversion in children. J Bone Joint Surg Br. 1981;63-B(2):198-208.
17. Volpon J, Avaliação e princípios do tratamento ortopédico do paciente com sequela de paralisia cerebral. Acta Ortop Bras. 1997;5(1):35-42.

18. Minear WL. A classification of cerebral palsy. Pediatrics. 1956;18(5):841-52.

19. Volpon JB. Orthopedic semiology. Rev Medicina, Ribeirão Preto.1996; 29(1/3): 67-79.

20. Fucs $P$ M, Svartman C, Kertzman P. Tratamento do quadril subluxado e luxado na paralisia cerebral. Revis Bras Ortop. 1998;33(1):15-9.

21. Bleck EE. Orthopaedic management in cerebral palsy, Philadelphia: MacKeith; 1987.

22. Howard CB, McKibbin B, Williams LA, Mackie I. Factors affecting the incidence of hip dislocation in cerebral palsy. J Bone Joint Surg Br. 1985;67(4):530-2.

23. Miller F, Cardoso Dias R, Dabney KW, Lipton GE, Triana M. Soft-tissue release for spastic hip subluxation in cerebral palsy. J Pediatr Orthop. 1997;17(5):571-84.

24. Tylkowski CM, Rosenthal RK, Simon SR. Proximal femoral osteotomy in cerebral palsy. Clin Orthop Relat Res. 1980;(151):183-92.

25. Phelps WM. Prevention of acquired dislocation of the hip in cerebral palsy. $J$ Bone Joint Surg Am. 1959;41(3):440-8.

26. Ferraretto I. Tratamento cirúrgico da paralisia cerebral. Rev Bras Ortop. 1971;6:37-40.

27. Carr C, Gage JR. The fate of the nonoperated hip in cerebral palsy. J Pediatr Orthop. 1987;7(3):262-7.

28. Cooperman DR, Bartucci E, Dietrick E, Millar EA. Hip dislocation in spastic cerebral palsy: long-term consequences. J Pediatr Orthop. 1987;7(3):268-76.

29. Reis HC, Hayakawa TP, Santos CA, Veloso SR, Bitar G. Cirurgia profilática de luxação do quadril em crianças com paralisia cerebral. Rev Bras Ortop. 1989;24:11-2.

30. Reimers J. Static and dynamic problems in spastic cerebral palsy. J Boné Joint Surg Br. 1973;55(4):822-7.

31. Bretas CT, Dias L. Rizotomia posterior seletiva em quadriplegia espástica. Rev Bras Ortop. 1994; 29(1):41-3.

32. Miller F, Bagg MR. Age and migration percentage as risk factors for progression in spastic hip disease. Dev Med Child Neurol. 1995;37(5):449-55.

33. Mubarak SJ, Valencia FG, Wenger DR. One-stage correction of the spastic dislocated hip. Use of pericapsular acetabuloplasty to improve coverage. J Boné Joint Surg Am. 1992;74(9):1347-57. 